\title{
AVALIAÇÃO DA CONDUTIVIDADE HIDRÁULICA EM DOIS USOS DO SOLO NA REGIÃO CENTRAL DO BRASIL
}

EVALUATION OF HYDRAULIC CONDUCTIVITY IN TWO LAND USES SOIL IN CENTRAL BRAZIL

\section{Miguel Angel Alfaro Soto}

Doutor em Geotecnia pela

Escola de Engenharia de São

Carlos, Universidade de São

Paulo (USP). Pesquisador no

Departamento de Geologia Aplicada

e Centro de Estudos Ambientais,

Universidade Estadual Paulista

"Júlio de Mesquita Filho" (UNESP) -

Rio Claro (SP), Brasil.

\section{Chang Hung Kiang}

Doutor em Geologia pela Universidade Northwestern. Professor no Departamento de Geologia Aplicada e Centro de Estudos Ambientais, UniversidadeEstadual Paulista "Júlio de Mesquita Filho" (UNESP) Rio Claro (SP), Brasil.

\section{Endereço para correspondência:}

Miguel Angel Alfaro SotoAvenida 24a, 1.515 - Bela Vista CEP 13506-900 - Rio Claro (SP), Brasil-E-mail: alfaro@rc.unesp.br

Recebido: $28 / 06 / 2016$

Aceito: 07/12/2017

\section{RESUMO}

Uma avaliação das condutividades hidráulicas em solos com tipos distintos de uso e ocupação - cultivo agrícola e cerrado nativo - foi realizada com a finalidade de verificar a interferência das intensas atividades agrícolas no processo de infiltração da água no solo e, consequentemente, recarga de aquíferos. Para determinação da condutividade hidráulica saturada em campo foram empregados permeâmetro Guelph e infiltrômetro de anel duplo. Os ensaios foram realizados em extensa área que abrange cinco estados na região central do Brasil, onde a atividade agrícola tem sido intensa. Avaliados mediante estatística paramétrica, os valores de condutividade hidráulica em áreas de cultivo mostraram-se estatisticamente diferentes e 4,5 vezes menores em relação aos obtidos em áreas de cerrado. Esses resultados indicam que as modificações da estrutura do solo, decorrentes das práticas de manejo para cultivo, afetam significativamente a condutividade hidráulica das porções superficiais e, portanto, a infiltração de água, responsável pela recarga dos aquíferos na região. Adicionalmente, verificou-se que os testes com infiltrômetros forneceram, em média, valores de condutividade estatisticamente diferentes e 2,2 vezes maiores do que os obtidos com permeâmetro Guelph.

Palavras-chave: áreas de cultivo; cerrado; permeâmetro Guelph; infiltrômetros de anel duplo.

\section{ABSTRACT}

Evaluation of the hydraulic conductivity in soil of different use - agricultural cultivation and undisturbed native Savannah - was performed, in order to verify the influence of intense agricultural activities on water infiltration in soils and, consequently, aquifer recharges. For this purpose, the experimental design involved the determination of saturated hydraulic conductivity using the Guelph permeameter and double-ring infiltrometer. Tests were performed on large area covering five states in Central Brazil, where the agriculture has been intense. Evaluated by parametric statistics, the hydraulic conductivity values in farms cultivated areas proved to be statistically different and 4.5 times lower compared to those ones obtained in savannah. These results showed that changes in soil structure resulting from management practices in crop areas significantly affect the hydraulic conductivity of the superficial portions and, therefore, the water infiltration, responsible for the aquifer recharge in that area. Additionally, it was found that the infiltrometer provided statistically different values of hydraulic conductivity and 2.2 times higher than those ones obtained with Guelph permeameter.

Keywords: crop areas; savannah; Guelph permeameter; double-ring infiltrometers. 


\section{INTRODUÇÃO}

O conhecimento da variabilidade das variáveis físico-hídricas do solo, no espaço e no tempo, é considerado, atualmente, o princípio básico para o manejo preciso das áreas agrícolas (JOSÉ et al., 2012).

Dentro dessas variáveis a condutividade hidráulica constitui o parâmetro mais importante que governa o movimento de água nos solos, e seu conhecimento é de grande importância em questões relacionadas à geotecnia, agronomia, hidrologia, contaminação e meio ambiente, entre outras áreas.

A medição da condutividade hidráulica pode ser realizada por meio de ensaios de laboratório e de campo. Neste, segundo Daniel (1989), podem ser realizados ensaios com permeâmetros de ponta porosa e de furos de sondagem, com drenos subterrâneos e de infiltração. No entanto, a dispersão dos resultados - provenientes dos diferentes métodos -, bem como a praticidade para execução dos testes, têm de ser levadas em consideração na escolha do equipamento.

Entre as técnicas disponíveis, os permeâmetros de furos de sondagem e ensaios de infiltração são os mais utilizados e difundidos (DANIEL, 1989).

Kanwar et al. (1989) compararam os resultados provenientes dos permeâmetros Guelph e de velocidade provenientes de um solo de till em lowa, Estados Unidos. No entanto, os diferentes métodos utilizados mostraram distintas tendências, conforme os tipos de solo e condições de campo.

Gupta et al. (1993) avaliaram o desempenho de infiltrômetro de anel duplo, simulador de chuvas, permeâmetro Guelph e este acoplado a um infiltrômetro, em Ottawa, Canadá. Os resultados provenientes do infiltrômetro de anel duplo e simulador de chuvas mostraram maiores valores médios de condutividade hidráulica e menores coeficientes de variação.

Verbist et al. (2013) compararam seis métodos (infiltrômetros simples e de duplo anel, infiltrômetro de carga constante, de trado inverso, infiltrômetro de tensão e simulador de chuvas) utilizados no semiárido no Chile. Alguns dos resultados mostraram diferenças com relação à condutividade hidráulica saturada $\left(K_{f s}\right)$, principalmente em função das diferentes técnicas de cálculo.
Esses estudos, entre outros encontrados na literatura (e.g., WANG et al., 2012; GHANI et al., 2013), foram realizados em solos com características próprias e diversas das localizadas no Brasil. Os solos brasileiros são, em grande parte, mais intemperizados, com sistemas heterogêneos de poros e distribuições de tamanho de poros multimodais, tal como mostrado por Alfaro Soto et al. (2015). A heterogeneidade de um sistema de poros pode ter origem na distribuição granulométrica específica ou na formação de porosidade secundária e, neste caso, está relacionada a processos de agregação física.

Solos com essas características podem apresentar peculiaridades, como valores de condutividade hidráulica mais altos (NOGAMI; VILLIBOUR, 1995) do que os encontrados em solos não intemperizados, em razão da elevada macroporosidade. Tal característica pode ser importante na hora de escolher o método de medição da condutividade hidráulica, visto que, segundo Lee (1983), a sua determinação possui um dos mais elevados coeficientes de variação (c.a. $200 \%$ ) em relação aos demais testes geotécnicos.

Além da variabilidade dos resultados decorrentes dos métodos de medição, é importante levar em consideração a provocada pela modificação antrópica do solo. Em estudo baseado no efeito decorrente de diferentes métodos de cultivo em um latossolo amarelo argiloso, Correia (1985) revelou que os sistemas de preparo do local estudado alteraram a densidade do solo em diferentes intensidades, porém com valores significativamente superiores em relação à floresta nativa. Resultados semelhantes foram verificados por Pires et al. (2012).

Entre os métodos expostos, o uso do permeâmetro Guelph tem se difundido em razão da praticidade (portabilidade e rapidez), substituindo testes comumente utilizados, tais como os de infiltração. No entanto, apesar de existirem pesquisas sobre resultados de comparação entre esses e outros experimentos, não são conhecidos resultados em solos com distribuição de tamanhos de poros multimodal - característica intrínseca de solos lateríticos - nem a relação entre solos inalterados e os modificados estruturalmente em razão das atividades decorrentes do cultivo. 
Nesse contexto, este trabalho teve como objetivo avaliar a influência de uso do solo, do ponto de vista hidráulico, quando utilizado no cultivo agrícola, com grande modificação antrópica do solo, sendo comparado com solos de cerrado com textura similar, porém sem modificação antrópica, ou seja, em estado de preservação natural.
Para determinação da condutividade hidráulica foram utilizados dois métodos: permeâmetro Guelph e infiltrômetro de anel duplo. Esses testes foram conduzidos em diferentes estados, como Bahia, Tocantins, Minas Gerais, Piauí e Goiás, sobre rochas do Grupo Urucuia, arcabouço geológico do Sistema Aquífero Urucuia.

\section{MATERIAIS E MÉTODOS}

Ao todo foram realizados 80 testes para obtenção da condutividade hidráulica saturada, sendo que $38-19 \mathrm{com}$ permeâmetro Guelph e 19 com infiltrômetros - em áreas agrícolas e $42-21$ com permeâmetro Guelph e 21 com infiltrômetros - em áreas de cerrado (não cultivadas). Cabe ressaltar que a identificação textural foi realizada por análise táctil visual. A Figura 1 mostra a localização dos testes realizados.

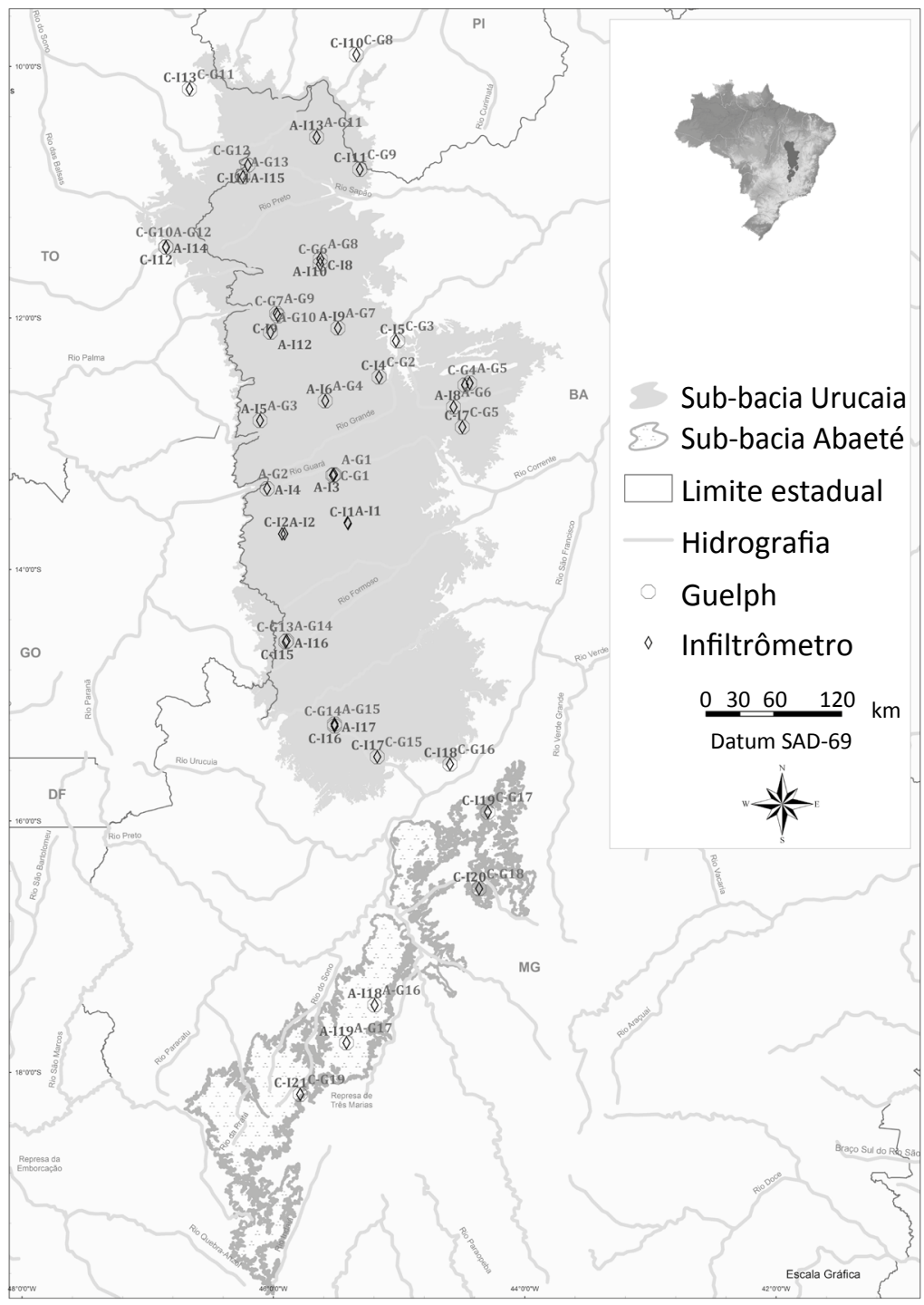

C: cerrados; A: áreas agrícolas; G: permeâmetro Guelph; I: infiltrômetros.

Figura 1 - Localização dos testes em cerrados e áreas agrícolas com permeâmetro Guelph e infiltrômetros. 


\section{Permeâmetro Guelph}

O permeâmetro Guelph funciona em regime de carga constante, sob o princípio do tubo de Mariotte. Os resultados obtidos por esse método são interpretados segundo o modelo teórico de Reynolds e Elrick (1985), baseado na equação de Richards para fluxo permanente em furo cilíndrico.

A equação para fluxo permanente é composta por duas parcelas, a primeira representa o fluxo saturado e a segunda o fluxo não saturado, dados pela Equação 1:

$Q=\left(\frac{2 \pi H^{2}}{C}+\pi a^{2}\right) K_{f s}+\left(\frac{2 \pi H}{C}\right) \phi_{G m}$

Em que:

$\mathrm{Q}\left[\mathrm{L}^{3} \mathrm{~T}^{-1}\right]=$ vazão em regime permanente;

$K_{f s}\left[\mathrm{LT}^{-1}\right]=$ condutividade hidráulica saturada de campo;

$\phi_{G m}\left[L^{2} T^{-1}\right]=$ potencial matricial de fluxo;

$\mathrm{H}[\mathrm{L}]=$ altura de carga hidráulica;

$\mathrm{a}[\mathrm{L}]=$ raio do furo no solo;

C [-] = parâmetro fator de forma, que depende da relação $\mathrm{H} / \mathrm{a}$ e do tipo de solo.

Graficamente, a Soilmoisture Equipment Corp. (1986) fornece o fator $C$ para três classes de solos (macroporosidade e textura).

A determinação dos parâmetros $K_{f s}$ e $f_{G m}$ da Equação 1 pode ser obtida, em geral, pelas técnicas (procedimentos de ensaio e cálculos) de uma e duas alturas de carga (REYNOLDS; ELRICK, 1985).

A primeira refere-se à aplicação de uma altura de carga hidráulica $\mathrm{H}$ constante até alcançar o regime perma- nente. Os parâmetros $K_{f s}$ e f $f_{G m}$ são determinados a partir das Equações 2 e 3 :

$K_{f s}=\frac{C Q}{2 \pi H^{2}+\pi a^{2} C+2 \pi H / \alpha}$

$\varphi_{G m}=\frac{C Q}{\left[\left(2 \pi H^{2}+\pi a^{2} C\right) \alpha+2 \pi H\right]}$

O parâmetro a (Equações 2 e 3) representa o grau de macroporosidade (fissuras no solo, formigueiros, cupinzeiros, furos causados por raízes, entre outros) e textura do solo. Para esse método, o parâmetro a é estimado a priori mediante avaliação visual. Os valores sugeridos estão resumidos no Quadro 1.

A técnica de duas alturas de cargas (ou mais de duas) consiste em aplicar duas ou mais cargas hidráulicas $\mathrm{H}_{\mathrm{i}}$ sucessivas. Depois de atingido o regime permanente e determinadas as vazões $Q_{i}$ e os parâmetros $C_{i}$ correspondentes a cada carga hidráulica, os parâmetros $K_{f s}$ e $f_{G m}$ são calculados a partir da solução de equações simultâneas (REYNOLDS; ELRICK, 1986), cuja solução é dada pelas Equações 4 e 5 :

$$
K_{f s}=\frac{\sum_{i=1}^{n} H_{i}^{2} \sum_{i=1}^{n} C_{i} Q_{i}\left(\frac{C_{i} \cdot a_{i}^{2}}{2}+H_{i}^{2}\right)-\sum_{i=1}^{n} H_{i} C_{i} Q_{i} \sum_{i=1}^{n} H_{i}\left(\frac{C_{i} a_{i}^{2}}{2}+H_{i}^{2}\right)}{2 \pi\left\{\sum_{i=1}^{n} H_{i}^{2} \sum_{i=1}^{n}\left(\frac{C_{i} a_{i}^{2}}{2}+H_{i}^{2}\right)^{2}-\left[\sum_{i=1}^{n} H_{i}^{2}\left(\frac{C_{i} a_{i}^{2}}{2}+H_{i}^{2}\right)\right]\right\}}
$$

$$
\phi_{G m}=\frac{\sum_{i=1}^{n} C_{i} Q_{i}\left|\frac{C_{i} \cdot a_{i}^{2}}{2}+H_{i}^{2}\right| \sum_{i=1}^{n} H_{i}\left|\frac{C_{i} \cdot a_{i}^{2}}{2}+H_{i}^{2}\right|-\sum_{i=1}^{n} H_{i} C_{i} Q_{i} \sum_{i=1}^{n}\left|\frac{C_{i} a_{i}^{2}}{2}+H_{i}^{2}\right|}{2 \pi\left\{\left|\sum_{i=1}^{n} H_{i}\right| \frac{C_{i} a_{i}^{2}}{2}+H_{i}^{2} \mid \|^{2}-\sum_{i=1}^{n} H_{i}^{2} \sum_{i=1}^{n}\left\langle\frac{C_{i} a_{i}^{2}}{2}+\left.H_{i}^{2}\right|^{2}\right\}\right.}
$$

\section{Quadro 1 - Valores a sugeridos por Reynolds e Elrick (1986).}

\begin{tabular}{|c|c|}
\hline$\alpha\left(\mathrm{cm}^{-1}\right)$ & Tipo de solo \\
0,01 & Argilas compactas (aterros, liners, sedimentos lacustres e marinhos). \\
0,04 & Solos de textura fina, principalmente sem macroporos e fissuras. \\
0,12 & Argilas até areias finas com alta a moderada quantidade de macroporos e fissuras. \\
0,36 & Areia grossa, incluindo solos com macroporos e fissuras. \\
\hline
\end{tabular}


Nesse caso, o parâmetro a é determinado por meio do ensaio, mediante a relação (Equação 6):

\section{Infiltrômetros de anel duplo}

O método do infiltrômetro de anel duplo é um dos mais tradicionais, sendo empregado em diferentes áreas de conhecimento, devido, provavelmente, aos procedimentos simples de ensaios, à fácil interpretação dos resultados e à maior representatividade (volume de solo ensaiado) em relação a testes em furo de sondagem.

Durante o processo de infiltração, a condutividade hidráulica saturada pode ser obtida tanto na carga constante como na carga variável. A Figura 2 mostra os parâmetros de medição para obtenção da condutividade hidráulica saturada, que pode ser calculada de duas maneiras:

Para carga constante (Equação 7):

$K_{f s}=Q /\left(A t .\left(H+Z_{w}\right) / Z_{w}\right)$

E para carga variável (Equação 8):

$K_{f s}=\left(Z_{w} \cdot \ln \left(H_{2} / H_{1}\right) / t\right)$

Em que:

$K_{f s}\left[\mathrm{LT}^{-1}\right]=$ condutividade hidráulica saturada de campo; $\mathrm{ZW}[\mathrm{L}]=$ profundidade da frente de saturação;

$A\left[L^{2}\right]=$ área transversal do anel;
$\alpha=K_{f s} / \phi_{G m}$

$t[T]=$ tempo entre duas leituras;

$\mathrm{Q}\left[\mathrm{L}^{3}\right]=$ volume de água infiltrada dentro do solo;

$\mathrm{H}[\mathrm{L}]=$ profundidade da água do anel quando é ensaiado a regime constante;

$\mathrm{H}_{1}$ e $\mathrm{H}_{2}[\mathrm{~L}]=$ profundidades inicial da água no anel e no tempo zero e " $\mathrm{t}$ ", respectivamente.

Os experimentos feitos utilizando o método do permeâmetro Guelph foram conduzidos pela técnica de uma altura de carga segundo procedimentos de testes e cálculos sugeridos por Reynolds e Elrick (1986), enquanto para o método do infiltrômetro foram usados os procedimentos contidos na norma ASTM D3385 (2008).

Tendo em vista a análise comparativa dos resultados entre os dois métodos, e considerando que o do infiltrômetro permite apenas ensaios em superfície, aqueles com o permeâmetro Guelph foram também realizados superficialmente (profundidade média de $30 \mathrm{~cm}$ ), porém evitando solos com elevada macroporosidade (e.g., presença de furos de raízes e de insetos), de forma a não fornecerem resultados incoerentes ou não representativos do local devido ao pequeno volume de água que este método proporciona.

\section{RESULTADOS E DISCUSSÃO}

A Tabela 1 reúne os resultados dos testes nas áreas de cultivo agrícola e cerrado, obtidos a partir de análises com o permeâmetro Guelph. Nessa tabela, são apresentados a condutividade hidráulica saturada (Kfs), o potencial matricial de fluxo $\left(f_{G m}\right)$ e o parâmetro a,bem como a média geométrica de cada um desses parâmetros.
A Tabela 2 agrupa os resultados dos testes nessas mesmas áreas, alcançados por meio de testes com infiltrômetro de anel duplo. Nessa tabela são apresentadas a condutividade hidráulica saturada (Kfs) e sua média geométrica.

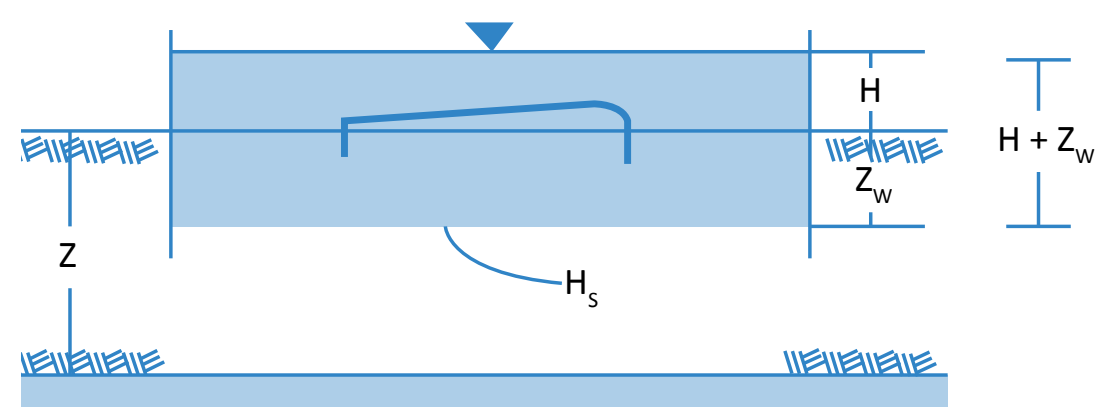

Figura 2 - Parâmetros para determinar a condutividade hidráulica saturada em infiltrômetros de anel duplo. 
Tabela 1 - Resultados de ensaios com o permeâmetro Guelph realizados em áreas de cultivo agrícola e cerrado.

\begin{tabular}{|c|c|c|c|c|c|}
\hline \multirow{2}{*}{$\begin{array}{l}\text { Ensaio } \\
\#\end{array}$} & \multicolumn{2}{|c|}{ Coordenadas } & \multirow{2}{*}{$\frac{K_{f s}}{\mathrm{~cm} / \mathrm{s}}$} & \multirow{2}{*}{$\frac{\phi_{\mathrm{m}}}{\mathrm{cm}^{2} / \mathrm{s}}$} & \multirow{2}{*}{$\frac{\alpha}{\mathrm{cm}^{-1}}$} \\
\hline & S & W & & & \\
\hline C1 & 13 ㅇ 38' 11,1" & $45 \circ 24^{\prime} 23,3^{\prime \prime}$ & $1,0 \mathrm{E}-02$ & 4,1E-02 & 0,250 \\
\hline $\mathrm{C} 2$ & $1343^{\prime} 01,4^{\prime \prime}$ & $4554^{\prime} 40,5^{\prime \prime}$ & $1,6 \mathrm{E}-02$ & $6,5 \mathrm{E}-02$ & 0,250 \\
\hline C3 & $13^{\circ} 15^{\prime} 02,9^{\prime \prime}$ & 45 o $31^{\prime} 36,4^{\prime \prime}$ & $4,7 \mathrm{E}-03$ & $1,9 \mathrm{E}-02$ & 0,250 \\
\hline C4 & $12 \div 28^{\prime} 10,7^{\prime \prime}$ & $4509^{\prime} 33,9^{\prime \prime}$ & $7,1 \mathrm{E}-03$ & $2,8 \mathrm{E}-02$ & 0,250 \\
\hline C5 & $12 \div 10^{\prime} 57,8^{\prime \prime}$ & $45^{\circ} 0,1^{\prime} 29,5^{\prime \prime}$ & $2,2 \mathrm{E}-04$ & 8,7E-04 & 0,250 \\
\hline C6 & $12 \circ 32^{\prime} 01,1^{\prime \prime}$ & $44^{\circ} 28^{\prime} 32,9^{\prime \prime}$ & $4,8 \mathrm{E}-03$ & $1,9 \mathrm{E}-02$ & 0,250 \\
\hline $\mathrm{C7}$ & $12^{\circ} 52^{\prime} 04,4^{\prime \prime}$ & $44 ㅇ 2^{\prime} 47,4^{\prime \prime}$ & $6,2 \mathrm{E}-03$ & $2,5 \mathrm{E}-02$ & 0,250 \\
\hline $\mathrm{C} 8$ & $11^{\circ} 34^{\prime} 16,3^{\prime \prime}$ & $45 \circ 37^{\prime} 43,0^{\prime \prime}$ & $6,2 \mathrm{E}-03$ & $2,5 \mathrm{E}-02$ & 0,250 \\
\hline C9 & $11059^{\prime} 01,6^{\prime \prime}$ & $4557^{\prime} 49,3^{\prime \prime}$ & $1,4 \mathrm{E}-02$ & $5,5 \mathrm{E}-02$ & 0,250 \\
\hline C10 & 9o $54^{\prime} 25,0^{\prime \prime}$ & $4520^{\prime} 22,1^{\prime \prime}$ & $7,2 \mathrm{E}-03$ & $2,9 \mathrm{E}-02$ & 0,250 \\
\hline C11 & $10 \circ 49^{\prime} 08,2^{\prime \prime}$ & $45 \circ 18^{\prime} 44,2^{\prime \prime}$ & $3,9 \mathrm{E}-03$ & $1,6 \mathrm{E}-02$ & 0,250 \\
\hline $\mathrm{C} 12$ & $11^{\circ} 26^{\prime} 11,6^{\prime \prime}$ & $46051^{\prime} 12,3^{\prime \prime}$ & $2,1 \mathrm{E}-02$ & $8,4 \mathrm{E}-02$ & 0,250 \\
\hline $\mathrm{C} 13$ & 10 일 $10^{\prime} 49,1^{\prime \prime}$ & $4639^{\prime} 59,9^{\prime \prime}$ & $6,1 \mathrm{E}-03$ & $2,4 \mathrm{E}-02$ & 0,250 \\
\hline C14 & $10 \cong 47^{\prime} 06,5^{\prime \prime}$ & $46012^{\prime} 07,4^{\prime \prime}$ & $2,1 \mathrm{E}-02$ & $8,4 \mathrm{E}-02$ & 0,250 \\
\hline C15 & $14^{\circ} 34^{\prime} 36,8^{\prime \prime}$ & $45 ㅇ 4^{\prime} 05,5^{\prime \prime}$ & $1,5 \mathrm{E}-02$ & $6,0 \mathrm{E}-02$ & 0,250 \\
\hline C16 & $15014^{\prime} 45,5^{\prime \prime}$ & $45 \div 30^{\prime} 42,2^{\prime \prime}$ & $4,0 \mathrm{E}-03$ & $1,6 \mathrm{E}-02$ & 0,250 \\
\hline C17 & 15 ㅇ $29^{\prime} 26,9^{\prime \prime}$ & $4510^{\prime} 23,1^{\prime \prime}$ & $4,8 \mathrm{E}-03$ & $1,9 \mathrm{E}-02$ & 0,250 \\
\hline $\mathrm{C} 18$ & 15 o $32^{\prime} 59,7 "$ & $44035^{\prime} 40,3^{\prime \prime}$ & $8,7 \mathrm{E}-03$ & $3,5 \mathrm{E}-02$ & 0,250 \\
\hline C19 & $15 \div 55^{\prime} 49,0^{\prime \prime}$ & $4417^{\prime} 32,8^{\prime \prime}$ & $1,8 \mathrm{E}-03$ & $9,5 \mathrm{E}-03$ & 0,190 \\
\hline $\mathrm{C} 20$ & $16 \div 32^{\prime} 25,0^{\prime \prime}$ & $44 ㅇ=21^{\prime} 45,8^{\prime \prime}$ & $2,7 \mathrm{E}-02$ & $8,9 \mathrm{E}-02$ & 0,300 \\
\hline C21 & 18 o $10^{\prime} 35,1^{\prime \prime}$ & $4547^{\prime} 12,6^{\prime \prime}$ & $2,1 \mathrm{E}-02$ & $8,4 \mathrm{E}-02$ & 0,250 \\
\hline Média & & & $7,0 \mathrm{E}-03$ & $2,8 \mathrm{E}-02$ & 0,250 \\
\hline A1 & $1337^{\prime} 41,9^{\prime \prime}$ & $45^{\circ} 24^{\prime} 32,5^{\prime \prime}$ & $1,9 \mathrm{E}-03$ & $9,4 \mathrm{E}-03$ & 0,200 \\
\hline$A 2$ & $1343^{\prime} 03,9^{\prime \prime}$ & 45 으 55' 53,2" & $1,8 \mathrm{E}-03$ & $1,5 \mathrm{E}-02$ & 0,120 \\
\hline$A 3$ & $1314^{\prime} 46,5^{\prime \prime}$ & $4531^{\prime} 00,9^{\prime \prime}$ & $1,4 \mathrm{E}-03$ & $1,1 \mathrm{E}-02$ & 0,120 \\
\hline A4 & $1321^{\prime} 31,0^{\prime \prime}$ & $46002^{\prime} 55,1^{\prime \prime}$ & $1,8 \mathrm{E}-03$ & 1,7E-02 & 0,100 \\
\hline A5 & $12 \circ 48^{\prime} 56,9^{\prime \prime}$ & $46006^{\prime} 22,2^{\prime \prime}$ & $2,1 \mathrm{E}-03$ & $1,8 \mathrm{E}-02$ & 0,120 \\
\hline A6 & 12 o $39^{\prime} 33,6^{\prime \prime}$ & 45 은 $35^{\prime} 13,2^{\prime \prime}$ & $1,5 \mathrm{E}-03$ & $9,0 \mathrm{E}-03$ & 0,170 \\
\hline A7 & 12 우 $31^{\prime} 04,3^{\prime \prime}$ & $44 ㅇ 6^{\prime} 19,1^{\prime \prime}$ & $9,9 \mathrm{E}-05$ & $2,4 \mathrm{E}-02$ & 0,004 \\
\hline A8 & $12 \circ 42^{\prime} 32,6^{\prime \prime}$ & $44033^{\prime} 57,4^{\prime \prime}$ & $1,3 \mathrm{E}-03$ & 1,1E-02 & 0,120 \\
\hline A9 & $12 \circ 04^{\prime} 50,9^{\prime \prime}$ & $45 \circ 29^{\prime} 07,5^{\prime \prime}$ & $1,4 \mathrm{E}-03$ & $1,2 \mathrm{E}-02$ & 0,120 \\
\hline A10 & 11 우 $31^{\prime} 49,7^{\prime \prime}$ & 45 ㅇ $37^{\prime} 34,9^{\prime \prime}$ & $1,7 \mathrm{E}-03$ & $5,1 \mathrm{E}-03$ & 0,320 \\
\hline A11 & 11 ㅇ 57' 53,5" & $4558^{\prime} 24,8^{\prime \prime}$ & $1,1 \mathrm{E}-03$ & 2,7E-02 & 0,040 \\
\hline A12 & 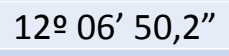 & $4601^{\prime} 23,1^{\prime \prime}$ & $1,1 \mathrm{E}-03$ & $4,1 \mathrm{E}-02$ & 0,030 \\
\hline A13 & $1033^{\prime} 42,9^{\prime \prime}$ & 45으 39' $19,7^{\prime \prime}$ & $9,5 \mathrm{E}-04$ & $7,9 \mathrm{E}-03$ & 0,120 \\
\hline A14 & $11026^{\prime} 08,6^{\prime \prime}$ & $4651^{\prime} 19,0^{\prime \prime}$ & $1,7 \mathrm{E}-03$ & $1,4 \mathrm{E}-02$ & 0,120 \\
\hline A15 & $1052^{\prime} 40,4^{\prime \prime}$ & $46014^{\prime} 29,1^{\prime \prime}$ & $1,8 \mathrm{E}-03$ & $1,5 \mathrm{E}-02$ & 0,120 \\
\hline A16 & $14 ㅇ 4^{\prime} 05,4^{\prime \prime}$ & $4553^{\prime} 36,2^{\prime \prime}$ & $2,5 \mathrm{E}-03$ & $2,1 \mathrm{E}-02$ & 0,120 \\
\hline A17 & 15 o $13^{\prime} 46,7^{\prime \prime}$ & $45 ㅇ 0^{\prime} 48,8^{\prime \prime}$ & $6,3 \mathrm{E}-03$ & $1,2 \mathrm{E}-02$ & 0,520 \\
\hline A18 & $17 ㅇ 27^{\prime} 55,8^{\prime \prime}$ & $4511^{\prime} 36,5^{\prime \prime}$ & $3,0 \mathrm{E}-03$ & $2,5 \mathrm{E}-02$ & 0,120 \\
\hline A19 & $17 ㅇ 5^{\prime} 57,0^{\prime \prime}$ & $4525^{\prime} 03,7^{\prime \prime}$ & $4,9 \mathrm{E}-03$ & $4,1 \mathrm{E}-02$ & 0,120 \\
\hline Média & & & $1,6 \mathrm{E}-03$ & $1,5 \mathrm{E}-02$ & 0,100 \\
\hline
\end{tabular}

C: cerrado; A: agrícola. 
Tabela 2 - Resultados de ensaios com infiltrômetros realizados em áreas de cultivo agrícola e cerrado.

\begin{tabular}{|c|c|c|c|}
\hline Ensaio & \multicolumn{2}{|c|}{ Coordenadas } & \multirow{2}{*}{$\frac{K_{f s}}{(\mathrm{~cm} / \mathrm{s})}$} \\
\hline$\#$ & $S$ & $\mathbf{W}$ & \\
\hline $\mathrm{C} 1$ & $13 ㅇ 8^{\prime} 11,1^{\prime \prime}$ & $45024^{\prime} 23,3^{\prime \prime}$ & $2,7 \mathrm{E}-02$ \\
\hline $\mathrm{C2}$ & 13 우 $43^{\prime} 01,4^{\prime \prime}$ & $45^{\circ}=54^{\prime} 40,5^{\prime \prime}$ & $5,6 \mathrm{E}-02$ \\
\hline C3 & 13 ㅇ $15^{\prime} 02,9^{\prime \prime}$ & $45031^{\prime} 36,4^{\prime \prime}$ & $1,3 \mathrm{E}-02$ \\
\hline C4 & $12 \div 28^{\prime} 10,7^{\prime \prime}$ & 45 잉 $33,9^{\prime \prime}$ & $1,2 \mathrm{E}-02$ \\
\hline C5 & $12 \div 10^{\prime} 57,8^{\prime \prime}$ & 45 잉 $01^{\prime} 29,5^{\prime \prime}$ & $2,1 \mathrm{E}-03$ \\
\hline C6 & 12 ㅇ $32^{\prime} 01,1^{\prime \prime}$ & $44^{\circ} 28^{\prime} 32,9^{\prime \prime}$ & $2,0 \mathrm{E}-02$ \\
\hline $\mathrm{C7}$ & $12^{\circ} 52^{\prime} 04,4^{\prime \prime}$ & $44029^{\prime} 47,4^{\prime \prime}$ & $1,4 \mathrm{E}-02$ \\
\hline $\mathrm{C} 8$ & 11 ㅇ $34^{\prime} 16,3^{\prime \prime}$ & $45037^{\prime} 43,0^{\prime \prime}$ & $3,9 \mathrm{E}-02$ \\
\hline $\mathrm{C} 9$ & 11 9 59' $01,6^{\prime \prime}$ & 45 ㅇ $57^{\prime} 49,3^{\prime \prime}$ & $2,4 \mathrm{E}-02$ \\
\hline C10 & 9o 54' $25,0^{\prime \prime}$ & 45 ㅇ $20^{\prime} 22,1^{\prime \prime}$ & $1,3 \mathrm{E}-02$ \\
\hline C11 & 10 영 $08,2^{\prime \prime}$ & $45^{\circ}=18^{\prime} 44,2^{\prime \prime}$ & $6,3 \mathrm{E}-03$ \\
\hline C12 & 11 은 $26^{\prime} 11,6^{\prime \prime}$ & $46 \div 51^{\prime} 12,3^{\prime \prime}$ & $1,8 \mathrm{E}-02$ \\
\hline $\mathrm{C} 13$ & 10 o $10^{\prime} 49,1^{\prime \prime}$ & $46039^{\prime} 59,9^{\prime \prime}$ & $8,2 \mathrm{E}-03$ \\
\hline C14 & $1047^{\prime} 06,5^{\prime \prime}$ & 46 ㅇ $12^{\prime} 07,4^{\prime \prime}$ & $2,6 \mathrm{E}-02$ \\
\hline C15 & $14 ㅇ=34^{\prime} 36,8^{\prime \prime}$ & $45^{\circ}=54^{\prime} 05,5^{\prime \prime}$ & $3,5 \mathrm{E}-02$ \\
\hline C16 & 15 o $14^{\prime} 45,5^{\prime \prime}$ & $45030^{\prime} 42,2^{\prime \prime}$ & $2,8 \mathrm{E}-02$ \\
\hline C17 & 15 을 $29^{\prime} 26,9^{\prime \prime}$ & 45 우 $10^{\prime} 23,1^{\prime \prime}$ & $1,7 \mathrm{E}-02$ \\
\hline C18 & $15^{\circ} 32^{\prime} 59,7^{\prime \prime}$ & $44035^{\prime} 40,3^{\prime \prime}$ & $8,8 \mathrm{E}-03$ \\
\hline C19 & $15055^{\prime} 49,0^{\prime \prime}$ & $44017^{\prime} 32,8^{\prime \prime}$ & $7,1 \mathrm{E}-03$ \\
\hline $\mathrm{C} 20$ & 16 o $32^{\prime} 25,0^{\prime \prime}$ & $44021^{\prime} 45,8^{\prime \prime}$ & $2,3 \mathrm{E}-02$ \\
\hline C21 & 18 ㅇ $10^{\prime} 35,1^{\prime \prime}$ & $4547^{\prime} 12,6^{\prime \prime}$ & $1,5 \mathrm{E}-02$ \\
\hline Média & & & $1,6 \mathrm{E}-02$ \\
\hline $\mathrm{A} 1$ & 13ㅇ 37" 41,9" & $45^{\circ}=24^{\prime} 32,5^{\prime \prime}$ & $9,5 \mathrm{E}-04$ \\
\hline$A 2$ & 13 엉 $03,9^{\prime \prime}$ & $45055^{\prime} 53,2^{\prime \prime}$ & $6,8 \mathrm{E}-03$ \\
\hline A3 & $1314^{\prime} 46,5^{\prime \prime}$ & 45 ㅇ $31^{\prime} 00,9^{\prime \prime}$ & $3,8 \mathrm{E}-03$ \\
\hline A4 & $1321^{\prime} 31,0^{\prime \prime}$ & $46002^{\prime} 55,1^{\prime \prime}$ & $5,0 \mathrm{E}-03$ \\
\hline A5 & $12 \circ 48^{\prime} 56,9^{\prime \prime}$ & $46 \div 06^{\prime} 22,2^{\prime \prime}$ & $3,1 \mathrm{E}-03$ \\
\hline A6 & $12 \div 39^{\prime} 33,6^{\prime \prime}$ & $45^{\circ}=35^{\prime} 13,2^{\prime \prime}$ & $9,1 \mathrm{E}-04$ \\
\hline A7 & 12 - $31^{\prime} 04,3^{\prime \prime}$ & $44026^{\prime} 19,1^{\prime \prime}$ & $1,6 \mathrm{E}-03$ \\
\hline A8 & $12 \circ 42^{\prime} 32,6^{\prime \prime}$ & $44033^{\prime} 57,4^{\prime \prime}$ & $1,7 \mathrm{E}-03$ \\
\hline A9 & 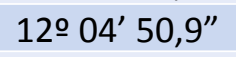 & $45 \circ 29^{\prime} 07,5^{\prime \prime}$ & $2,8 \mathrm{E}-03$ \\
\hline A10 & 11 익 $31^{\prime} 49,7^{\prime \prime}$ & $45 \div 37^{\prime} 34,9^{\prime \prime}$ & $2,2 \mathrm{E}-03$ \\
\hline A11 & 11 은 $53,5^{\prime \prime}$ & $45058^{\prime} 24,8^{\prime \prime}$ & $3,1 \mathrm{E}-03$ \\
\hline A12 & $12 \circ 06^{\prime} 50,2^{\prime \prime}$ & $46 \div 01^{\prime} 23,1^{\prime \prime}$ & $3,7 \mathrm{E}-03$ \\
\hline A13 & 10 우 $33^{\prime} 42,9^{\prime \prime}$ & 45 ㅇ $39^{\prime} 19,7^{\prime \prime}$ & $2,4 \mathrm{E}-03$ \\
\hline A14 & 11 우의 $08,6^{\prime \prime}$ & $46 \div 51^{\prime} 19,0^{\prime \prime}$ & $6,8 \mathrm{E}-03$ \\
\hline A15 & $1052^{\prime} 40,4^{\prime \prime}$ & $46014^{\prime} 29,1^{\prime \prime}$ & $3,3 \mathrm{E}-04$ \\
\hline A16 & $14 \div 34^{\prime} 05,4^{\prime \prime}$ & 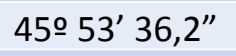 & 8,1E-03 \\
\hline A17 & 15 ㅇ $13^{\prime} 46,7^{\prime \prime}$ & 45 ㅇ $30^{\prime} 48,8^{\prime \prime}$ & $2,2 \mathrm{E}-02$ \\
\hline A18 & $17027^{\prime} 55,8^{\prime \prime}$ & 45 ㅇ $11^{\prime} 36,5^{\prime \prime}$ & $3,3 \mathrm{E}-02$ \\
\hline A19 & $17045^{\prime} 57,0^{\prime \prime}$ & 45 ㅇ $25^{\prime} 03,7^{\prime \prime}$ & $8,6 \mathrm{E}-03$ \\
\hline Média & & - & $3,4 \mathrm{E}-03$ \\
\hline
\end{tabular}

C: cerrado; A: agrícola. 
Esses resultados evidenciam diferenças tanto na comparação de valores médios entre métodos de ensaios (permeâmetro Guelph e infiltrômetros) como entre tipos de solo segundo seu uso e ocupação (solos de cultivo agrícola e cerrado). Para uma comparação quantitativa utilizando esses conjuntos de dados, foram realizados testes estatísticos de hipótese nula ( $\mathrm{Ho}$ ). A hipótese Ho considera que as médias das duas populações são iguais, enquanto a hipótese alternativa H1 considera-as diferentes. As populações analisadas nessas comparações foram:

- Os resultados dos dois métodos de campo (permeâmetro Guelph e infiltrômetros);

- Os resultados quanto aos tipos de uso do solo (cultivo agrícola e cerrado).

As análises estatísticas para verificação da Ho foram os testes $t\left(t_{1}\right.$ ou $t_{2}$, dependendo da igualdade ou diferença dos desvios padrões segundo teste $F$ ) e o teste $\mathrm{F}$, necessário para verificar a igualdade dos desvios padrões da variância. Esses testes são os mais recomendados devido ao número reduzido de amostras e porque apenas os desvios padrões amostrais são conhecidos. A estatística paramétrica foi possível pelo emprego do logaritmo natural dos dados amostrais de cada população, uma vez que esses elementos apresentam distribuição log-normal (típica de populações compostas por dados de condutividade hidráulica saturada).
A Tabela 3 resume os resultados desses testes de hipóteses, os quais são interpretados a partir de $P$, que representa a probabilidade de errar ou aceitar o resultado observado como válido. Assim, ao testar uma hipótese nula para um determinado nível de significância estipulado para o estudo $(a=5 \%)$, a hipótese será aceita se a estiver contido nesse intervalo de probabilidade (ou seja, P>a ), caso contrário, Ho será rejeitada.

Os valores $\mathrm{P}$ obtidos pelo teste $t$ Student são menores do que o nível de significância de $5 \%$ em todos os casos. A hipótese nula que afirma igualdade de médias (entre métodos ou tipos de uso de solo) é, portanto, rejeitada, passando a validar a hipótese alternativa (H1). Consequentemente, os métodos Guelph e infiltrômetro produzem resultados de condutividade hidráulica estatisticamente diferentes. Essa afirmação é válida para todos os dados $(P=0,04)$, apenas para os dos ensaios em cultivo agrícola $(\mathrm{P}=0,02)$ e para aqueles provenientes de cerrado $(P=0,06)$.

Um confronto entre os dados experimentais obtidos por ambos os métodos pode ser observado na Figura 3, na qual os resultados obtidos pelo permeâmetro Guelph são menores do que os do infiltrômetro. A partir desses resultados verifica-se que a relação entre condutividades hidráulicas médias $K_{f s}$ (Infiltrômetro) $/ K_{f s}$ (Guelph) é igual a 2,2.

Tabela 3 - Resultados dos testes de hipóteses para comparação de valores médios de $K_{f s}$ provenientes de diferentes métodos (Guelph e infiltrômetros) e tipos de uso do solo (cultivo agrícola e cerrado).

\begin{tabular}{|c|c|c|c|c|c|c|}
\hline \multirow{4}{*}{ Dados } & \multicolumn{4}{|c|}{ Comparações } & \multirow{3}{*}{\multicolumn{2}{|c|}{$\begin{array}{c}\text { Valores P } \\
\text { Testes }\end{array}$}} \\
\hline & \multirow{2}{*}{\multicolumn{2}{|c|}{$\begin{array}{c}\text { Entre métodos } \\
K_{f s} \text { médio }\end{array}$}} & \multirow{2}{*}{\multicolumn{2}{|c|}{$\begin{array}{c}\text { Entre tipos de uso do solo } \\
\qquad K_{f s} \text { médio }\end{array}$}} & & \\
\hline & & & & & & \\
\hline & Guelph & Infiltrômetro & Agrícola & Cerrado & $F^{*}$ & $\mathrm{~T}^{* *}$ \\
\hline Agrícola & $1,6 \mathrm{E}-03$ & $3,4 \mathrm{E}-03$ & - & - & 0,146 & 0,020 \\
\hline Cerrado & $7,0 \mathrm{E}-03$ & $1,6 \mathrm{E}-02$ & - & - & 0,395 & 0,006 \\
\hline Guelph & - & - & $1,6 \mathrm{E}-03$ & $7,0 \mathrm{E}-03$ & 0,297 & 0,000 \\
\hline Infiltrômetro & - & - & $3,4 \mathrm{E}-03$ & $1,6 \mathrm{E}-02$ & 0,184 & 0,000 \\
\hline
\end{tabular}

$\mathrm{t}: \mathrm{t}_{1}$, se $\alpha(\mathrm{F}) \geq 0 ; 05$; caso contrário, $\mathrm{t}_{2} ;{ }^{*}$ Ho aceita para $\alpha=5 \% ; *{ }^{*} 1$ aceita para $\alpha=5 \%$. 
Este valor deve estar relacionado ao menor volume de amostra ocupada durante a infiltração, uma vez considerados os efeitos capilares em sua formulação e pelo fato de serem evitadas zonas de macroporos se comparado com o infiltrômetro. Trabalhos como os de Mohanty et al. (1994) e Gintanau (2011) têm mostrado que, entre os métodos de campo, os infiltrômetros apresentam sempre os valores mais altos de condutividade hidráulica, em razão do maior volume de solo ocupado durante o ensaio.

De forma análoga, a Tabela 3 mostra que a comparação dos resultados de condutividade hidráulica obtidos para cada solo (cerrado e cultivo agrícola), pelo método do permeâmetro Guelph (valor $\mathrm{P}=0$ para o teste $t$ ) ou de infiltrômetros (valor $\mathrm{P}=0$ para o teste $t$ ), produz resultados estatisticamente diferentes.

A Figura 4 apresenta os valores de $K_{f s,}$ estatisticamente, e os valores médios e dispersão dos resultados provenientes de ambos os métodos, em forma de box-plot, para solos de cerrado e cultivo agrícola. Esses resultados exibem relações entre condutividades hidráulicas médias $K_{f s}$ (cerrado)/ $K_{f s}$ (agrícola) iguais a 4,4 e 4,6, fruto dos testes com permeâmetro Guelph e infiltrômetros, respectivamente.

Como pode ser observado na Figura 4, é evidente a superioridade dos valores de Kfs obtidos para as áreas de cerrado em relação às de cultivo agrícola, o que é

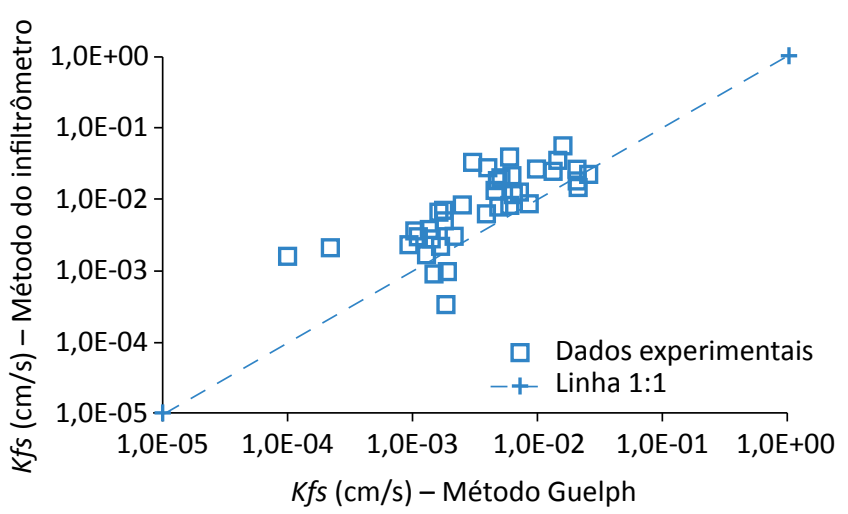

Figura 3 - Confronto de resultados de $K_{f s}$ entre métodos de medição. coerente com o fato de serem áreas de preservação e, portanto, inalteradas pela ação antrópica - e por apresentarem maior macroporosidade em razão da interferência biológica (raízes e atividade de insetos formigas, cupins etc.). Ao contrário do cerrado, os solos das áreas de cultivo agrícola apresentam intensa alteração antrópica, com Kfs menores - sejam medidas pelo método Guelph ou por infiltrômetros - em razão da maior densidade ocasionada pelas práticas de manejo e cultivo.

Os resultados da Figura 4 são similares aos encontrados por Silva et al. (2014), utilizando infiltrômetros de anel duplo em solos de textura média na cidade de Rio Verde, Goiás, onde foi observada a redução de $K_{f s}$ de solos em área de cultivo em torno de quatro vezes em relação a solos de cerrados.

Testes semelhantes foram realizados por Viana e Donagemma (2016), porém utilizando o permeâmetro Guelph em solos arenosos na Chapada Gaúcha, Minas Gerais, e em Campo Verde, Mato Grosso. Nesse estudo se observou redução de $K_{f s}$ de solos em área de cultivo entre quatro a oito vezes em relação a solos de cerrados. No entanto, testes em solos de textura argilosa, como os realizados por Batista e Sousa (2015) na cidade de Iporá, Goiás, mostraram diferenças de resultados do ponto de vista estatístico.

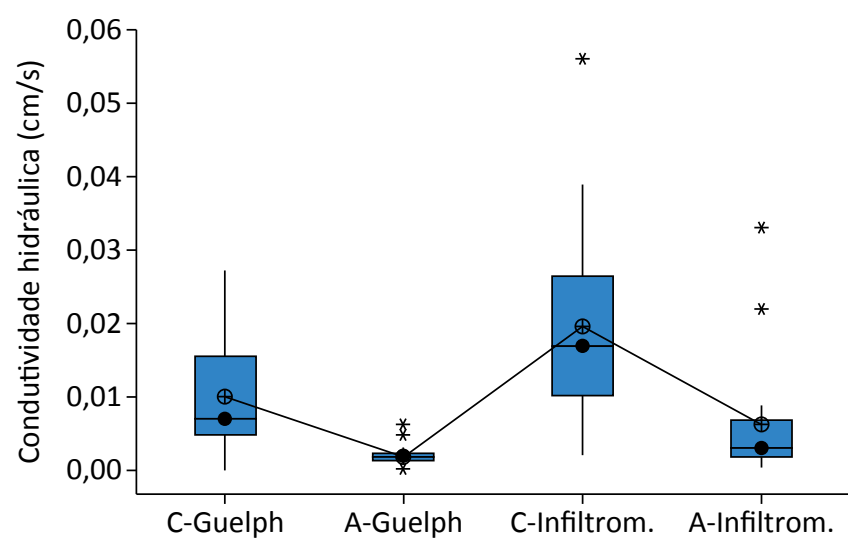

Figura 4 - Confronto de resultados de $K_{f s}$ (box-plot) provenientes de solos com diferentes tipos de uso (cerrado e cultivo agrícola). 


\section{CONCLUSÕES}

A avaliação da influência do uso do solo em extensa área do território brasileiro, do ponto de vista hidráulico, utilizando os métodos do permeâmetro Guelph e do infiltrômetro de anel duplo, apresentou resultados que devem ser considerados na análise da recarga do aquífero.

Observou-se que a condutividade hidráulica em solos inalterados (cerrado) apresentou valores em média 4,5 vezes maiores do que em solos com intensa atividade antrópica (cultivo agrícola). A análise estatística comparativa determinou que as condutividades hidráulicas médias desses solos (independentemente do método de medição utilizado) são estatisticamente diferentes para o nível de significância de 5\%. Do ponto de vista geotécnico, esses solos com distinto uso apresentam semelhante característica textural, porém diferem em densidade e macroporosidade decorrentes das práticas de manejo para cultivo. Esses resultados sugerem a intensa utilização agrícola de solos, que interfere de maneira significativa no processo de infiltração de águas, prejudicando a recarga de aquíferos. Tal constatação é preocupante, haja vista tratar-se de importante fronteira agrícola em expansão no país, implantada em área de exposição do Aquífero Urucuia, e que utiliza águas deste sistema em grande escala.

De forma similar, a análise estatística comparativa entre os resultados de condutividade hidráulica obtidos pelos métodos Guelph e de infiltrômetro mostrou que, para os tipos de solos analisados (cerrado inalterado e cultivado), os resultados são estatisticamente diferentes para o nível de significância de $5 \%$. As condutividades hidráulicas resultantes dos ensaios com infiltrômetro de anel duplo são, em média, 2,2 vezes maiores do que as obtidas com o permeâmetro Guelph, reforçando a hipótese de que os ensaios de anel duplo sofrem maior influência da macroporosidade do solo por ensaiar uma área superficial maior do que o método Guelph.

\section{REFERÊNCIAS}

ALFARO SOTO, M. A.; BASSO, J. B.; CHANG, H. K.; VAN GENUCHTEN, M. T. Simulação de fluxo e transporte de íons de vinhaça através de vertente da formação Rio Claro. Revista Brasileira de Águas Subterrâneas, v. 29, n. 2, p. 162-174, 2015.

AMERICAN SOCIETY FOR TESTING AND MATERIALS (ASTM). ASTM D3385. Standard test method for infiltration rate of soils in field using double-ring infiltrometer. Estados Unidos: ASTM, 2008. 8p.

BATISTA, D. F.; SOUSA, F. A. Avaliação da condutividade hidráulica do solo sobre condições de cobertura por cerrado e pastagem. Revista Eletrônica do Curso de Geografia, n. 25, 2015. Disponível em: <https://revistas.ufg.br/geoambiente/ article/view/35332/0>. Acesso em: jan. 2016.

CORRÊA, J. C. Efeito de métodos de cultivo em algumas propriedades físicas de um Latossolo Amarelo muito argiloso do Estado do Amazonas. Pesquisa Agropecuária Brasileira, v. 20, p. 1317-1322, 1985.

DANIEL, D. E. In situ Hydraulic Test for Compacted Clay. Journal of Geotechnical Engineering, v. 115, n. 9, p. 1205-1226, 1989.

GHANI, F.; TABATABAEI, S. H.; SHAYANNEJAD, M.; GHORBANI DASHTAKI, S. H. Comparison of four in-situ methods for measuring saturated hydraulic conductivity. Water Engineering, v. 5, n. 15, p. 57-68, 2013.

GINTANAU, S. H. Application of Guelph permeameter and double-ring infiltrometer for determination offield permeability values. 71f. Monografia (Bacharelado em Engenharia Civil) - Universidade de Tecnologia da Malásia, 2011.

GUPTA, R. K.; RUDRA, R. P.; DICKINSON, W. T.; PATNI, N. K.; WALL, G. J. Comparison of saturated hydraulic conductivity measured by various held methods. Transactions of the ASAE, v. 36, n. 1, p. 51-55, 1993.

JOSÉ, J. V.; REZENDE, R.; MARQUES, P. A. A.; GONÇALVES, A. C. A.; SOUZA, R. S. Variabilidade espacial de variáveis físicohídricas de dois latossolos da região noroeste do estado do Paraná. Irriga, Botucatu, v. 17, n. 2, p. 208-219, abr.-jun. 2012. 
KANWAR, R. S.; RIZVI, H. A.; AHMED, M.; HORTON JR., R.; MARLEY. S. J. Measurement of field-saturated hydraulic conductivity by using Guelph and velocity permeameters. Transactions of the ASAE, v. 32, p. 1885-1890, 1990.

LEE, I. K.; WHITE, W.; INGLES, O. G. Geotechnical Engineering. Estados Unidos: Pitman Publishing, 1983. 508 p. Cap 2.

MOHANTY, B. P.; KANWAR, R. S.; EVERTS, C. J. Comparison of saturated hydraulic conductivity measurement methods for a glacial-till soil. Soil Science Society of America Journal, v. 58, p. 672-677, 1994.

NOGAMI, J. S.; VILLIBOR, D. F. Pavimentação de baixo custo com solos lateríticos. São Paulo: Villibor, 1995. 213p.

PIRES, B. S.; DIAS JUNIOR, M. S.; ROCHA, W. W.; ARAÚJO JÚNIOR, C. F.; CARVALHO, R. C. R. Modelos de capacidade de suporte de carga de um latossolo vermelho-amarelo sob diferentes usos e manejos. Revista Brasileira de Ciência do Solo, v. 36, n. 2, p. 635-642, 2012.

REYNOLDS, W. D.; ELRICK, D. E. A method for simultaneous in situ measurement in the vadose zone of field saturated hydraulic conductivity, sorptivity and the conductivity-pressure head relationships. Ground Water Monitoring Review, v. 6, n. 1, p. 84-95, 1986.

REYNOLDS, W. D.; ELRICK, D. E. In situ measurement of field saturated hydraulic conductivity, sorptivity and the $\alpha$-parameter using the Guelph permeameter. Soil Science, v. 140, n. 4, p. 292-302, 1985.

SILVA, N. F.; CUNHA, F. N.; OLIVEIRA, R. C.; CABRAL, F. R.; TEIXEIRA, M. B.; CARVALHO, J. J. Características físico-hídricas de um latossolo sob diferentes sistemas de manejo. Revista Brasileira de Agricultura Irrigada, v. 8, n. 5, p. 375-390, 2014.

SOILMOISTURE EQUIPMENT CORP. Guelph Permeameter. Operating - Instructions, Soilmoisture manual. Estados Unidos: Soilmoisture Equipment Corp., 1986. 25p.

VERBIST, K.; CORNELIS, W. M.; TORFS, S.; GABRIELS, D. Comparing methods to determine hydraulic conductivities on stony soils. Soil Science Society of America Journal, v. 77, p. 25-42, 2013.

VIANA, J. H. M.; DONAGEMMA, G. K. O solo sob ameaça: conexões necessárias ao manejo e conservação do solo e água. In: REUNIÃO BRASILEIRA DE MANEJO E CONSERVAÇÃO DO SOLO E DA ÁGUA, Foz do Iguaçu. Anais... Curitiba: SBCS; Londrina: IAPAR, 2016.

WANG, H.; SONG, S.; TANG, X. Comparison of determination methods for saturated soil hydraulic conductivity with Guelph infiltrometer. Nongye Gongcheng Xuebao. Transactions of the Chinese Society of Agricultural Engineering, v. 28, n. 24, p. 99-104, 2012. 